\title{
Deep learning-based framework for the distinction of membranous nephropathy: a new approach through hyperspectral imagery
}

Tianqi Tu

Peking University China-Japan School of Clinical Medicine

\section{Xueling Wei}

Beijing University of Chemical Technology

\section{Yue Yang}

China-Japan Friendship Hospital

\section{Nianrong Zhang}

China-Japan Friendship Hospital

\section{Wei Li}

Beijing Institute of Technology

Xiaowen Tu ( $\nabla$ xiaowentu@126.com )

Department of Nephrology, PLA Rocket Force Characteristic Medical Center, Beijing, China https://orcid.org/0000-0002-2364-0110

\section{Wenge Li}

China-Japan Friendship Hospital

\section{Research article}

Keywords: membranous nephropathy, idiopathic membranous nephropathy, hepatitis B virus, hyperspectral imagery, deep learning

Posted Date: June 9th, 2020

DOI: https://doi.org/10.21203/rs.3.rs-30329/v1

License: (c) (i) This work is licensed under a Creative Commons Attribution 4.0 International License. Read Full License

Version of Record: A version of this preprint was published at BMC Nephrology on June 19th, 2021. See the published version at https://doi.org/10.1186/s12882-021-02421-y. 


\section{Abstract}

Background Common subtypes seen in Chinese patients with membranous nephropathy (MN) include idiopathic membranous nephropathy (IMN) and hepatitis B virus-related membranous nephropathy (HBV$\mathrm{MN})$. However, in some cases, the morphologic differences are not visible under the light microscope in the renal biopsy tissue.

Methods $₫$ We proposed a deep learning-based framework for processing hyperspectral images of renal biopsy tissue to define the difference between IMN and HBV-MN based on the component of their immune complex deposition.

Results: The proposed framework can achieve an overall accuracy of $95.04 \%$ for multiclass classification, which also proven to obtain a better performance compared to the support vector machine (SVM)-based algorithms.

ConclusionХIMN and HBV-MN could be correctly separated via the deep learning framework using hyperspectral imagery. Our results suggest the potential of the deep learning algorithm as a new method to aid the diagnosis process of $\mathrm{MN}$.

\section{Background}

Membranous nephropathy ( $\mathrm{MN}$ ) is one of the most common causes of nephrotic syndrome in adult patients across all ethnicities, which also serves significantly to end-stage renal disease ${ }^{[1-3]}$. Up-to-date data confirmed that MN makes up approximately $20-40 \%$ among all patients with nephrotic syndrome worldwide ${ }^{[4,5]}$. Most cases among MN are idiopathic membranous nephropathy (IMN), and the rest are secondary membranous nephropathy (SMN) attributed to various causes like lupus erythematosus (SLE), malignancy, hepatitis B virus infection. ${ }^{[6]}$ An estimated more than 257 million individuals worldwide are suffering from chronic HBV infection, leading to nearly one million deaths annually ${ }^{[7]}$. HBV infection is prevalent among the Chinese population; approximately $20 \%$ of patients with HBV infection develop extrahepatic manifestations ${ }^{[8]}$. Among the extrahepatic manifestations related to HBV infection, HBVrelated membranous nephropathy is a common outcome of HBV infection. In the diagnosis process of $\mathrm{MN}$, the most crucial part is to distinguish between idiopathic or secondary based on the patients' laboratory examination and renal biopsy outcome, which determines the specific treatment decisions of the two as well as guides the long-term evaluating prognosis.

Conventional approaches in detecting glomerular hepatitis antigen/antibody consist of: positive immunofluorescence outcome and specific pathological features found in renal biopsy tissue using a light microscope and electron microscope. Theoretically, patients with IMN were characterized by glomerular lesions with only immune complex deposited under the epithelial and thickening glomerular basement membrane, and patients with HBV-MN were associated with cell proliferation and multiple locations immune complex deposition in addition to the glomerular basement membrane lesions. ${ }^{[1]}$ 
However, in our previous laboratory practice, we have noticed that methods based on immunochemistry have a certain percentage of false positives, not to mention pathological features between IMN and HBV$\mathrm{MN}$ on the conventional bi-dimensional image obtained by light microscope are highly identical.

Since the morphological distinction between IMN and HBV-MN is subtle, an alternative method apart from current ones is much needed. In this study, we proposed a computer-based automatic identification method using a hyperspectral microscopy system, which proved can be a sufficient supplementary approach to separate IMN and HBV-MN.

Superior to classic imaging techniques which only provides spatial information, hyperspectral imagery (HSI) is an advanced imaging technique capable of obtaining both spatial and spectral information of the target material. ${ }^{[9,10]}$ Every pixel of a hyperspectral image carries a specific value called spectral signature determined by the material being observed, which can be extracted by deep learning algorithms to analyze. IMN is a single-organ autoimmune disease, the component of its immune complex (IC) deposition is connected to IgG4 initiation in pathogenesis ${ }^{[11]}$, where components of HBV-MN deposition are intimately related to hepatitis B virion. The different pathogenesis of IMN and HBV-MN provides us the possibility to identify their distinction using a hyperspectral imaging system. As an emerging imaging technique for medical applications, $\mathrm{HSI}$ also offers excellent potential for noninvasive disease diagnosis and surgical guidance. ${ }^{[9]}$

This study is the first time that the hyperspectral characteristics are applied for differing IMN from HBVMN in adult patients with membranous nephropathy, after analyzing each patients' hyperspectral image, their distinction is straight forward.

\section{Methods}

We retrieved 20 patients diagnosed with membranous nephropathy by clinical data and renal biopsy in China-Japan Friendship Hospital from July 2019 and September 2019, which includes 10 IMN patients and $10 \mathrm{HBV}-\mathrm{MN}$ patients. Inclusion criteria: IMN group: MN patients with unclear etiology and glomerular lesions limited to only immune complex deposited under the epithelial and thickening glomerular basement membrane; HBV-MN group: (1) serum HBV markers positive; (2) excluded other causes attribute to secondary renal disease (lupus erythematosus, drugs, toxins, other infections, or malignancy) (3) presence of detectable HBV-related antigen or antibody in renal biopsy tissue; Also (3) is the most fundamental and indispensable rule of all those listed above. In all cases, MN accompanied by other pathological patterns, for instance, diabetic nephropathy, IgA nephropathy, were ruled out.

\subsection{Sample Description}

We achieved the process of collecting glomerular characteristics for hyperspectral analysis by using routinely processed renal biopsy tissue. Each sample was stained with hematoxylin-eosin (HE), periodic acid-Schiff (PAS), Masson's trichrome, and Jones's silver. All renal tissue samples were observed by light and immunofluorescence microscopy beforehand, and all patients' diagnosis was confirmed based on 
current criteria. Later an experienced expert re-examined these biopsies, and all patients were eligible for consideration.

\subsection{Hyperspectral Image Collection}

We performed the hyperspectral imaging using a compound microscope system, where a SOC-710 portable hyperspectral imager is attached with a light microscope to obtain the HS images in the spectral range comprised between 400-1000 nm. For each patient, we randomly chose 2-3 glomeruli per slide, then manually marked out every immune complex in the subepithelial area using ENVI 14.0 for further analysis.

\subsection{Image De-noising}

Unprocessed HSI data usually contains high spectral noise generated by the imaging system, which can lead to undesirable effects. In this step, the de-noising process is achieved following equation (1). The concept of $D(i, j)$ is to replace the center pixel with the average value of all pixels inside the local window where $\mathrm{W}$ and $\mathrm{H}$ represent the width and height of the filtering window, respectively.

$$
D(i, j)=\sum_{(m, n) \in R, H} S(m, n) / H W
$$

Fig. 1 shows a glomerulus from the $10^{\text {th }}$ channel of an HBV-MN patients' HS image before and after the de-noising technique. The remarkable improvement confirms the effect of reducing the system noise of HSI data.

\subsection{Projection Transformation}

As aforementioned, HSI data possessed hundreds of spectral channels information, hence a mustrequired step for utilizing HSI data is to reduce the redundant information in its spectral signature. Projection transformation is an advanced method for acquiring maximum reduced subspace of target without losing its essential information. Current projection transformation techniques include principal component analysis (PCA) and independent component analysis (ICA) and Fisher's linear discriminant analysis (LDA). However, a significant drawback of those techniques is that all of them are legitimate only when target data is subordinated to gaussian distribution. In this study, we were able to develop an alternative method named local Fisher's discriminant analysis (LFDA) that integrates the advantages of both Fisher's linear discriminant analysis (LDA) and locality-preserving projections (LPP), meanwhile bypassing the limitation of gaussian distribution. ${ }^{[12-15]}$

\subsection{Proposed Deep Learning Framework}

Following the image de-noising and projection transformation procedures mentioned above, we constructed a deep neural network (DNN) to extract and classify the intrinsic and high-level features of 
the different glomerular images ${ }^{[16]}$. To be specific, support vector machine (SVM), extreme learning machine (ELM) ${ }^{[17]}$, Alexnet ${ }^{[18]}$, Resnet20 ${ }^{[19]}$ and VGG19 ${ }^{[20]}$ were implied on the MN database with and without the pre-processing procedures to achieve the ultimate goal of formulating an $\mathrm{MN}$ identification architecture that can automatically distinguish HBV-MN from IMN.

In this paper, we applied DNN to identifying glomerular disease in microscopic hyperspectral images for the first time, providing verification and supplementation for the outcome of immunofluorescence or light microscope.

\section{Results}

Based on the LDFA-DNN method mentioned above, we achieved the comparative evaluation of the hyperspectral image data of HBV-MN with IMN from renal biopsy tissue. We collected $30 \mathrm{HBV}-\mathrm{MN}$ images and $24 \mathrm{IMN}$ images from 10 patients of each case for the MN database. The size of each hyperspectral image is $696 \times 520$, with 128 channels of spectral dimension. Fig.2 (a) and (c) shown an HBV-MN and an IMN sample under the light microscope, in (b) (c) we marked out their immune complexes in the foreground, respectively. Fig. 3 elaborates its corresponding immune complex deposition under an electron microscope. The side-by-side images of HBV-MN and IMN showed a high similarity of their biological features, whether under light or electron microscope. Hence we proceed with the pre-processing chain of de-noising and projection transformation to extract the intrinsic feature for further classification.

After LFDA is applied, we conducted experiments to investigate the performance of VGG networks under different dimensions of the reduced subspace. Table 1 shows that LFDA obtains the optimal classification performance for VGG networks when the dimension is 9.After de-noising all the original hyperspectral images, LFDA is applied to convert the massive HSI data into a reduced subspace. In Fig.4, (a) and (b) are the before and after projection transformation feature distribution of one testing sample. The results exhibit the effectiveness of LFDA for seeking a subspace with maximum separability for features.

Table 1. Comparisons with different dimensions of reduced subspace for LFDA.

\begin{tabular}{llllll} 
metrics & $\mathbf{5}$ & $\mathbf{9}$ & $\mathbf{1 3}$ & $\mathbf{1 7}$ & $\mathbf{2 1}$ \\
\hline OA (\%) & 93.17 & 95.04 & 91.87 & 92.27 & 92.95 \\
Kappa & 0.8629 & 0.9006 & 0.8367 & 0.8458 & 0.8590
\end{tabular}

We also validated several patch sizes for the VGG network because of the significant impact it may cause on the performance of DNNs. The results in Table 2 suggest $11 \times 11$ is the optimal size of patches.

Table 2. The classification performance of various patch sizes. 


\begin{tabular}{lllll} 
metrics & $\mathbf{9 \times 9}$ & $\mathbf{1 1 \times 1 1}$ & $\mathbf{1 3} \times \mathbf{1 3}$ & $\mathbf{1 5 \times 1 5}$ \\
\hline $\mathrm{OA}(\%)$ & 93.13 & 95.04 & 92.43 & 94.36 \\
Kappa & 0.8629 & 0.9006 & 0.8485 & 0.8872
\end{tabular}

For identifying HBV-MN and IMN, the database for the training and testing process are strictly separated, hence guaranteed the eligibility and consistency of comparing the performance between each method.

Table 3 presented the experiment performance of classifying HBV-MN and IMN through various approaches. Overall accuracy (OA), average accuracy (AA), and Kappa Coefficient were used to assess the result of different algorithms. In this study, the proposed framework improves the overall accuracy to $95.04 \%$ ( $12 \%$ more than the overall accuracy obtained by algorithms without pre-processing chain). Fig.5 shows the complete performance results obtained by each approach. Compared to conventional machine learning algorithms, deep learning models offers a better performance distinguishing HBV-MN from IMN. Furthermore, adding the de-noising and projection transformation boosts LFDA-DNNs' performance significantly, hence validating the necessity of the pre-processing chain.

Table 3. The classification performance of the LFDA-DNN using the MN dataset.

\begin{tabular}{llllll} 
Comparisons & HBV-MN & IMN & OA (\%) & AA (\%) & Kappa \\
\hline SVM & 65.20 & 68.27 & 66.80 & 66.74 & 0.3347 \\
\hline ELM & 61.85 & 71.62 & 66.94 & 66.74 & 0.3356 \\
\hline Alexnet & 65.16 & 69.01 & 67.16 & 67.09 & 0.3418 \\
\hline Resnet20 & 80.17 & 68.31 & 73.99 & 74.24 & 0.4819 \\
\hline VGG19 & 79.70 & 85.72 & 82.84 & 82.71 & 0.6554 \\
\hline LFDA-SVM & 94.28 & 88.35 & 91.19 & 91.31 & 0.8239 \\
\hline LFDA-ELM & 94.92 & 84.58 & 89.54 & 89.75 & 0.7913 \\
\hline LFDA-Alexnet & 92.00 & 96.00 & 94.09 & 94.00 & 0.8814 \\
\hline LFDA-Resnet20 & 96.88 & 91.72 & 94.19 & 94.30 & 0.8839 \\
\hline LFDA-VGG19 & 95.67 & 94.45 & 95.04 & 95.06 & 0.9006
\end{tabular}


Fig. 6 shows that in this study, LFDA-VGG19 achieves outstanding performance for separate HBV-MN from IMN. Its overall accuracy improves by $3.85 \%$ and $5.50 \%$ higher in comparison with LFDA-SVM and LFDA-ELM, respectively. However, while LFDA-Resnet20 maintains the best one-on-one classification result for HBV-MN and LFDA-Alexnet for IMN, LFDA-VGG19 presents a more stable and balanced performance to identify and distinguish the two. Therefore LFDA-VGG19 is chosen as the ideal deep learning framework for this study.

For further research purposes, we also added 10 HBV-MN patients with negative serum HBV markers into the framework to compare with previous HBV-MN patients, and the sorting result came back as low separability between serum HBV markers positive HBV-MN patients and those without serum antigens/antibodies. This suggests that the hepatitis B virus could cause extrahepatic immune complex formation/deposition without presenting serological evidence.

\section{Discussion}

WHO estimates that more than 257 million persons or $3.5 \%$ of the world population were living with chronic HBV infection ${ }^{[21]}$. Hepatitis B virus infection is a significant health problem in China. Previous cases reports have associated chronic hepatitis B virus (HBV) infection with several types of glomerulonephritis (GN). Almost all morphological forms of renal disease, including membranoproliferative GN(MPGN) ${ }^{[22]}$, mesangial proliferative GN, minimal change disease, focal glomerulosclerosis, and IgA nephropathy (IgAN) have been described ${ }^{23]}$. The most common type of GN is membranous nephropathy, and the natural history of this condition is not clear ${ }^{[24]}$. By employing a hyperspectral imaging-based architecture consists of filtering, projection transformation, high-level feature extractor, and softmax classifier, we were able to identify HBV-MN and IMN cases using a noninvasive new technique.

Compared to conventional bi-dimensional images obtained by light microscopy, the hyperspectral imaging system provides superior resolution and sensitivity as well as its capability to process massamount multi-dimensional images simultaneously $[25,26]$. HSI technique was applied fields such as remote sensing field ${ }^{[27]}$, archeology $[28,29]$, drug identification ${ }^{[30,31]}$, among many others. Throughout the years, its importance in biomedical fields has evolved gradually. In theory, compositions of different materials will present significant discrepancies in spectral curves [10]; therefore, curves of IMN and HBV$\mathrm{MN}$ would be ready to distinguish due to their different immune complex components led by diverse pathogenesis. The hyperspectral analysis method we presented has the potential to demonstrates a wide spectral range and a high spectral resolution. Additionally, it enables the fast acquisition of a large number of hyperspectral images and data, and in combination with de-noising procedure ensures accuracy and reproducible imaging spectroscopy and spectrometry ${ }^{[9]}$.

Up to date medical hyperspectral imaging has been employed in several different areas such as blood

vessel visualization enhancement ${ }^{[32]}$, estimation of the cholesterol levels ${ }^{[33]}$, histopathological tissue 
analysis ${ }^{[34,35]}$, identification of glioblastoma tumor ${ }^{[36]}$. At the same time, there are no studies regarding membranous nephropathy and especially the distinction between IMN and HBV-MN.

\section{Conclusion}

In this study, we first propose the hyperspectral analysis as a new method for the characterization and distinction of HBV-MN from IMN, especially for cases where the discrimination is not ideal with the light microscopy. The hyperspectral imaging-based LFDA-DNN architecture could serve as an alternative method to the identification of HBV-MN as a result of its superiority in projection transformation and data classification, and it also indicated its nonnegligible potential for further application in the medical field.

\section{Abbreviations}

MN: membranous nephropathy; IMN: idiopathic membranous nephropathy; HBV: hepatitis B virus; DL: deep learning; HSI: hyperspectral imaging; DNN: deep neural network; LFDA: local Fisher's discriminant analysis; SMV: support vector machine $\mathbb{E L M} \otimes$ extreme learning machine

\section{Declarations}

Acknowledgments

The authors are grateful to all the participants.

Authors' contributions

WGL and WL designed the study. TQT and XLW collected the data, interpreted the statistical results, and wrote the first draft. YY and NRZ performed the histological examination of the kidney. XWT and WGL revised the manuscript. All authors read and approved the final manuscript.

Funding

The study was conducted with the help of China-Japan Friendship Hospital research expense. The grants played a role in the design of the study, collection of data, interpretation of data, and in writing the manuscript.

Availability of data and materials

The dataset used and/or analyzed during the current study are available from the corresponding author on reasonable request.

Ethics approval and consent to participate

This study was approved through the local ethics committee of China-Japan Friendship Hospital. The study has been performed in accordance with the ethical standards laid down in the 1964 Declaration of 
Helsinki. All patients gave written informed consent before data collection.

Consent for publication

Not applicable.

Competing interests

The authors declare that they have no competing interests.

\section{References}

1. Jiang Z, Cai M, Dong B, Yan Y, Yang B, Wang M, Wang Y, Li X, Lian L, Li S, Zuo L. Clinicopathological features of atypical membranous nephropathy with unknown etiology in adult Chinese patients. Medicine (Baltimore). 2018. 97(32): e11608.

2. Cattran DC, Brenchley PE. Membranous nephropathy: integrating basic science into improved clinical management. Kidney Int. 2017. 91(3): 566-574.

3. Couser WG. Primary Membranous Nephropathy. Clin J Am Soc Nephrol. 2017. 12(6): 983-997.

4. Tang L, Yao J, Kong X, Sun Q, Wang Z, Zhang Y, Wang P, Liu Y, Li W, Cui M, Zhen J, Xu D. Increasing prevalence of membranous nephropathy in patients with primary glomerular diseases: A crosssectional study in China. Nephrology (Carlton). 2017. 22(2): 168-173.

5. Xu X, Wang G, Chen N, Lu T, Nie S, Xu G, Zhang P, Luo Y, Wang Y, Wang X, Schwartz J, Geng J, Hou FF. Long-Term Exposure to Air Pollution and Increased Risk of Membranous Nephropathy in China. J Am Soc Nephrol. 2016. 27(12): 3739-3746.

6. Ronco P, Debiec H. Pathophysiological advances in membranous nephropathy: time for a shift in patient's care. Lancet. 2015. 385(9981): 1983-92.

7. Schweitzer A, Horn J, Mikolajczyk RT, Krause G, Ott JJ. Estimations of the worldwide prevalence of chronic hepatitis B virus infection: a systematic review of data published between 1965 and 2013. Lancet. 2015. 386(10003): 1546-55.

8. Li P, Wei RB, Tang L, Wu J, Zhang XG, Chen XM. Clinical and pathological analysis of hepatitis $B$ virus-related membranous nephropathy and idiopathic membranous nephropathy. Clin Nephrol. 2012. 78(6): 456-64.

9. Lu G, Fei B. Medical hyperspectral imaging: a review. J Biomed Opt. 2014. 19(1): 10901.

10. Halicek M, Fabelo H, Ortega S, Callico GM, Fei B. In-Vivo and Ex-Vivo Tissue Analysis through Hyperspectral Imaging Techniques: Revealing the Invisible Features of Cancer. Cancers (Basel). 2019. 11(6).

11. Filippone EJ. Idiopathic membranous nephropathy and IgG4: an interesting relationship. Clin Nephrol. 2014. 82(1): 7-15.

12. Wang R, Nie F, Hong R, Chang X, Yang X, Yu W. Fast and Orthogonal Locality Preserving Projections for Dimensionality Reduction. IEEE Trans Image Process. 2017. 26(10): 5019-5030. 
13. Zhu X, Qiu H. High Accuracy Human Activity Recognition Based on Sparse Locality Preserving Projections. PLoS One. 2016. 11(11): e0166567.

14. Jia P, Huang T, Wang L, Duan S, Yan J, Wang L. A Novel Pre-Processing Technique for Original Feature Matrix of Electronic Nose Based on Supervised Locality Preserving Projections. Sensors (Basel). 2016. 16(7).

15. Zhong F, Zhang J, Li D. Discriminant locality preserving projections based on L1-norm maximization. IEEE Trans Neural Netw Learn Syst. 2014. 25(11): 2065-74.

16. Wei X, Tu T, Zhang N, et al. Membranous Nephropathy Identification Using Hyperspectral Microscopic Images[C]//Chinese Conference on Pattern Recognition and Computer Vision (PRCV). Springer, Cham, 2019: 173-184.

17. GB Huang HZ, Ding X. Extreme learning machine for regression and multiclass classification. IEEE Transactions on Systems, Man, and Cybernetics, part B (Cybernetics). 2011. 42(2): 513-529.

18. A Krizhevsky IS, Hinton GE. Imagenet classification with deep convolutional neural networks. International Conference on Neutral Information Processing Systems. 2012.

19. $\mathrm{K}$ He XZ, S Ren JS. Deep residual learning for image recognition. Proceedings of the IEEE conference on computer vision and pattern recognition. 2016: 770-778.

20. K Simonyan AZ. Very deep convolutional networks for large-scale image recognition. arXiv preprint arXiv:1409.1556. 2014.

21. Hepatitis B World Health Organization Fact Sheet. 2019. https://www.who.int/en/news-room/factsheets/detail/hepatitis-b. \accessed on 28 February 2020》

22. Iatropoulos P, Daina E, Curreri M, Piras R, Valoti E, Mele C, Bresin E, Gamba S, Alberti M, Breno M, Perna A, Bettoni S, Sabadini E, Murer L, Vivarelli M, Noris M, Remuzzi G, Registry of Membranoproliferative Glomerulonephritis/C3 Glomerulopathy, Nastasi. Cluster Analysis Identifies Distinct Pathogenetic Patterns in C3 Glomerulopathies/Immune Complex-Mediated Membranoproliferative GN. J Am Soc Nephrol. 2018. 29(1): 283-294.

23. Sethi S, Fervenza FC. Standardized classification and reporting of glomerulonephritis. Nephrol Dial Transplant. 2019. 34(2): 193-199.

24. Seitz-Polski B, Lambeau G, Esnault V. [Membranous nephropathy: Pathophysiology and natural history]. Nephrol Ther. 2017. 13 Suppl 1: S75-S81.

25. W Li GW, Du Q. Transferred deep learning for anomaly detection in hyperspectral imagery. IEEE Geoscience and Remote Sensing Letters. 2017. 14(5): 597-561.

26. M Zhang WL, Du Q. Diverse region-based CNN for hyperspectral image classification. IEEE Trans Image Process. 2018.

27. Van der Meer F D VdWHMA, Van Ruitenbeek F J A ea. Multi-and hyperspectral geologic remote sensing: A review. International Journal of Applied Earth Observation and Geoinformation. 2012. 14(1): 112-128. 
28. Liang $\mathrm{H}$. Advances in multispectral and hyperspectral imaging for archaeology and art conservation. Applied Physics A. 2012.

29. V Miljković DG. Adaptation of industrial hyperspectral line scanner for archaeological applications. International Archives of the Photogrammetry, Remote Sensing and Spatial Information Sciences. 2016. 5.

30. WF de Carvalho Rocha GPS, Março PH. Quantitative analysis of piroxicam polymorphs pharmaceutical mixtures by hyperspectral imaging and chemometrics. Chemometr Intell Lab Syst. 2011. 106(2): 198-204.

31. França Lde M, Pimentel MF, Simões Sda S, Grangeiro S Jr, Prats-Montalbán JM, Ferrer A. NIR hyperspectral imaging to evaluate degradation in captopril commercial tablets. Eur J Pharm Biopharm. 2016. 104: 180-8.

32. Bjorgan A, Denstedt M, Milani `c M, Paluchowski LA, Randeberg LL. Vessel contrast enhancement in hyperspectral images. Optical Biopsy XIII: Toward Real-Time Spectroscopic Imaging and Diagnosis. 2015.

33. Milanic M, Bjorgan A, Larsson M, Strömberg T, Randeberg LL. Detection of hypercholesterolemia using hyperspectral imaging of human skin. Clinical and Biomedical Spectroscopy and Imaging IV. 2015.

34. Ortega S, Fabelo H, Camacho R, de la Luz Plaza M, Callicó GM, Sarmiento R. Detecting brain tumor in pathological slides using hyperspectral imaging. Biomed Opt Express. 2018. 9(2): 818-831.

35. Lu C, Mandal M. Toward automatic mitotic cell detection and segmentation in multispectral histopathological images. IEEE J Biomed Health Inform. 2014. 18(2): 594-605.

36. Fabelo H, Halicek M, Ortega S, Shahedi M, Szolna A, Piñeiro JF, Sosa C, O'Shanahan AJ, Bisshopp S, Espino C, Márquez M, Hernández M, Carrera D, Morera J, Callico GM, Sarmiento R, Fei B. Deep Learning-Based Framework for In Vivo Identification of Glioblastoma Tumor using Hyperspectral Images of Human Brain. Sensors (Basel). 2019. 19(4).

\section{Figures}




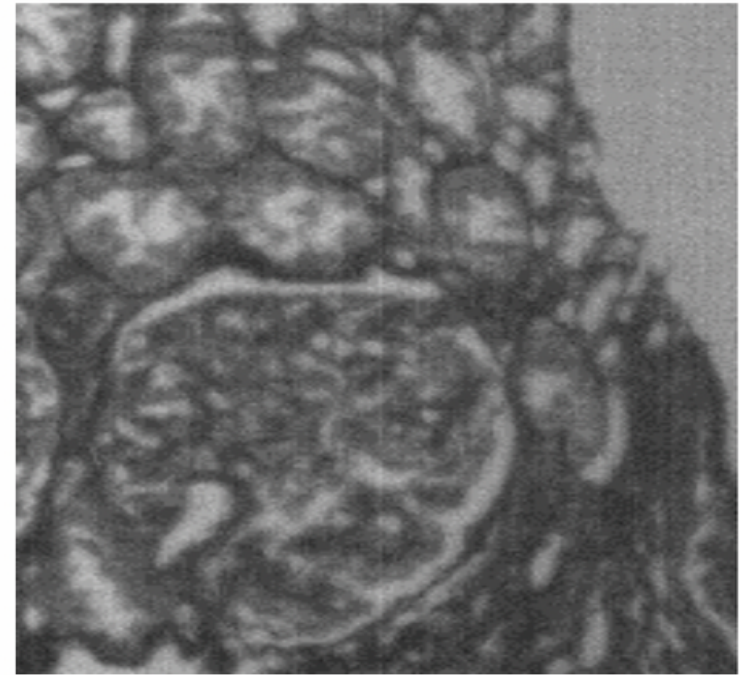

(a)

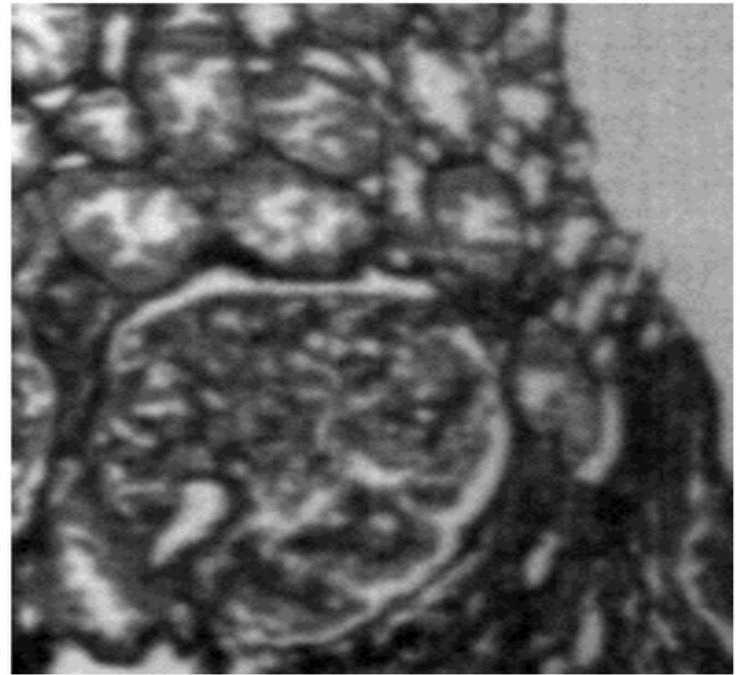

(b)

Figure 1

(a) and (b) are the before and after image de-noising of an HBV-MN patient's glomeruli.

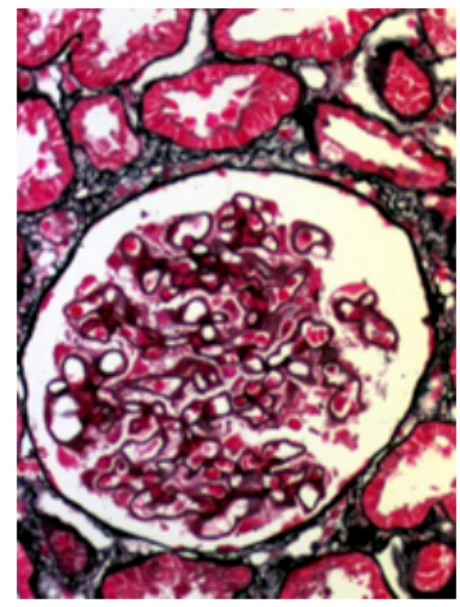

(a)

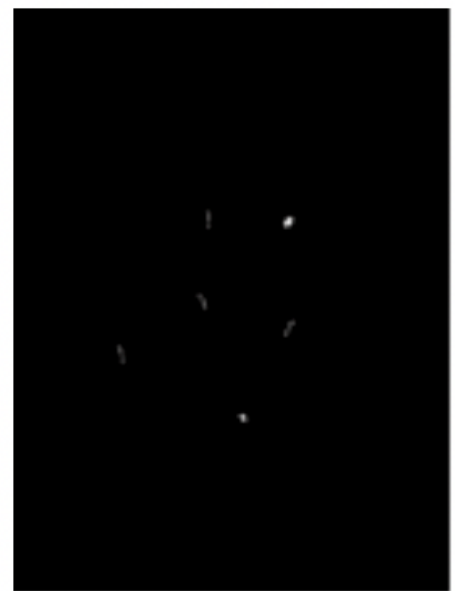

(b)

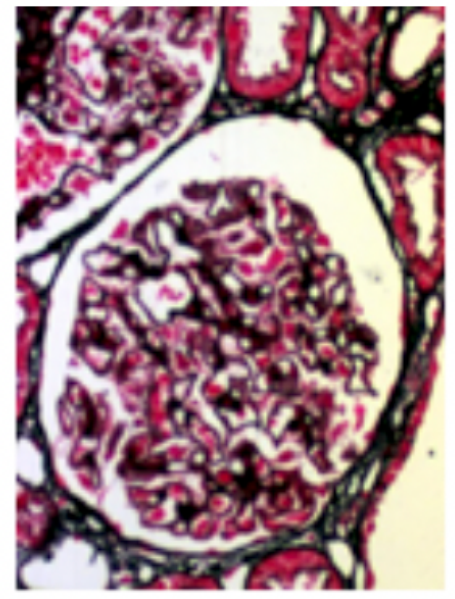

(c)

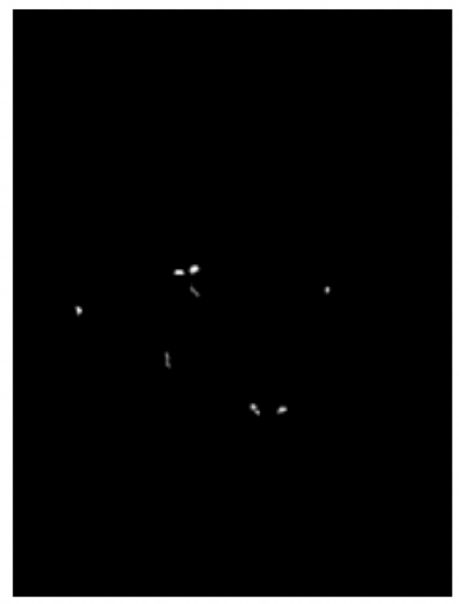

(d)

Figure 2

(a) and (c) are the image of an HBV-MN and IMN glomeruli; (b) and (d) are the corresponding ground truth maps with white pixels represents the marked out immune complexes. 


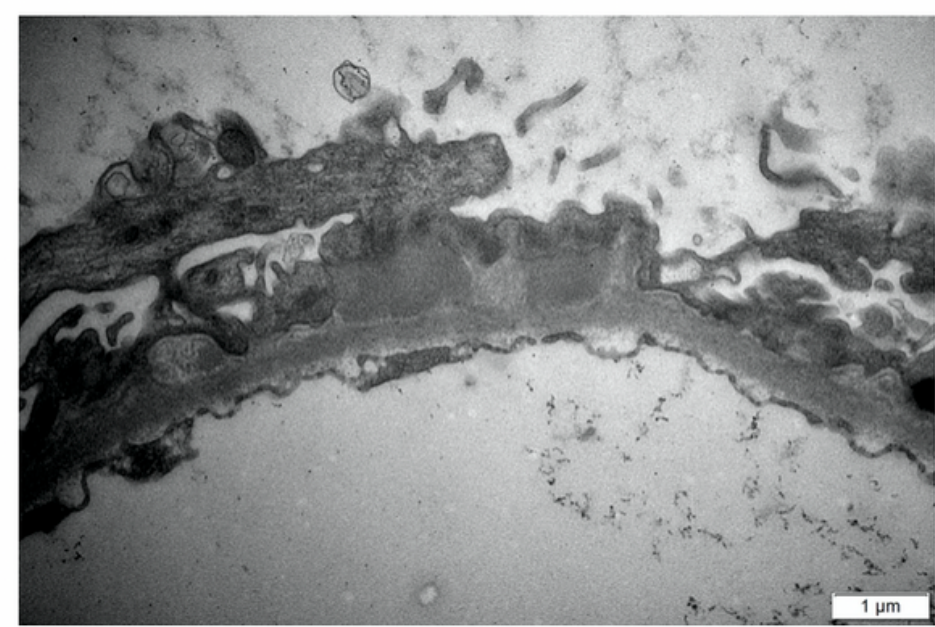

(a)

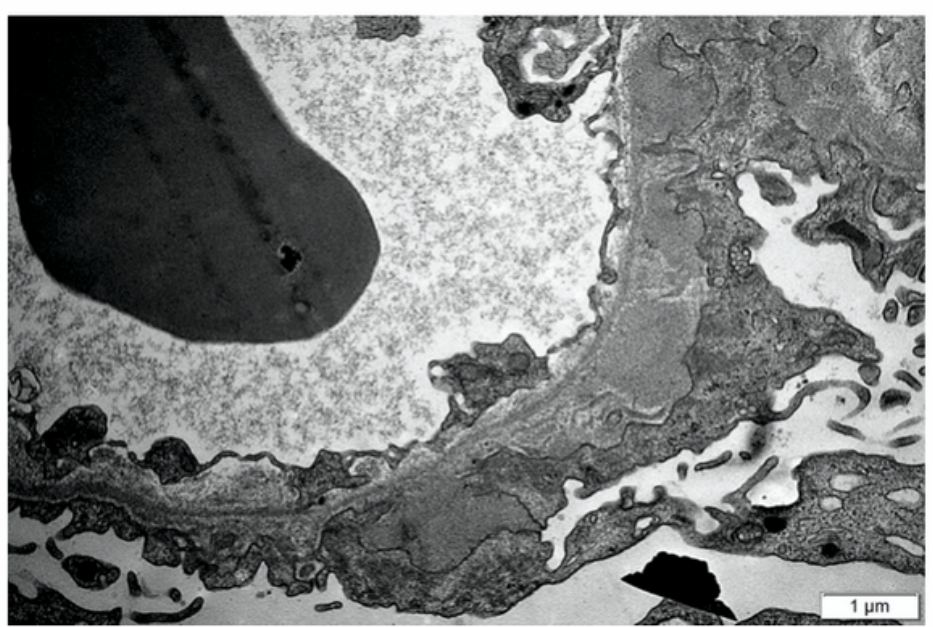

(b)

\section{Figure 3}

(a) and (b) are the pictures of an HBV-MN and an IMN immune complex deposition under $\times 20,000$ electron microscope.

Source data.

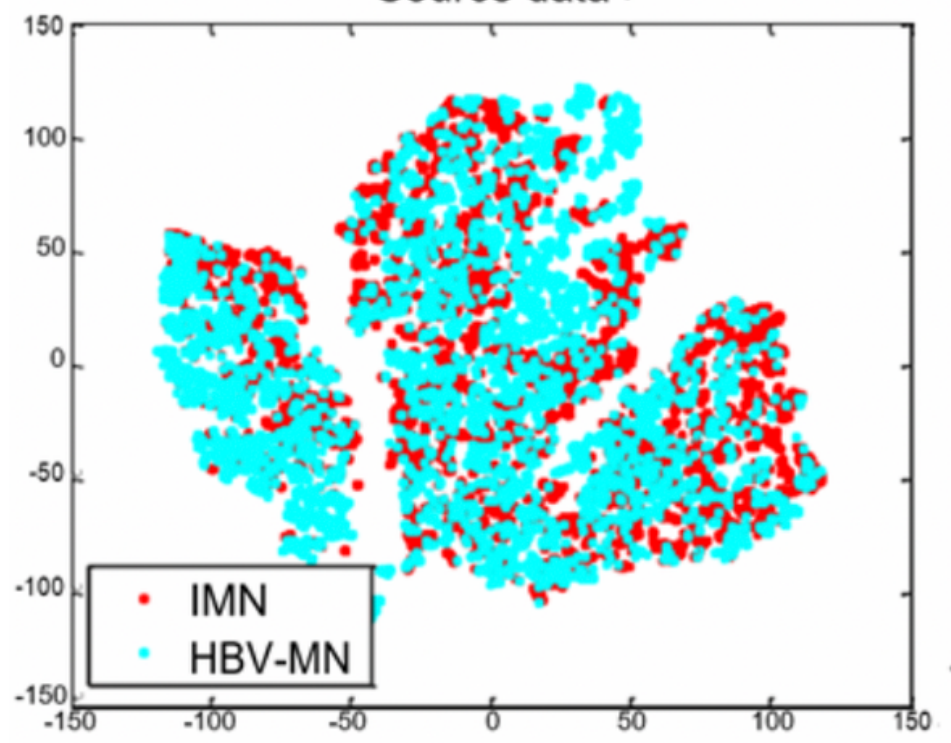

(a)
Features .

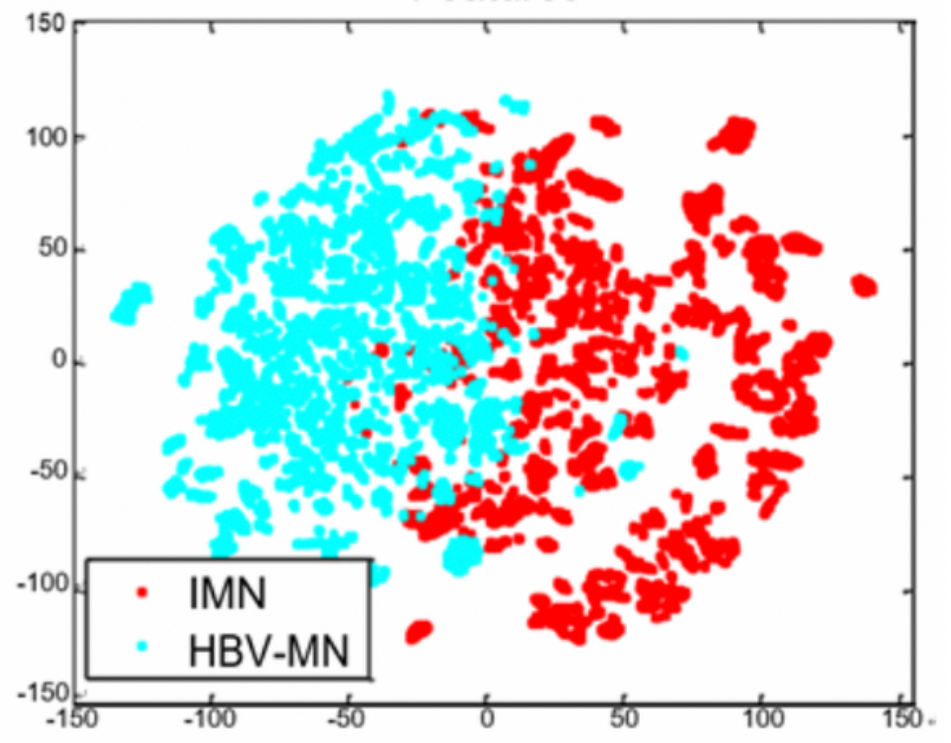

(b) .

Figure 4

(a) and (b) presents the distribution of samples' intrinsic features before and after the projection procedure. 


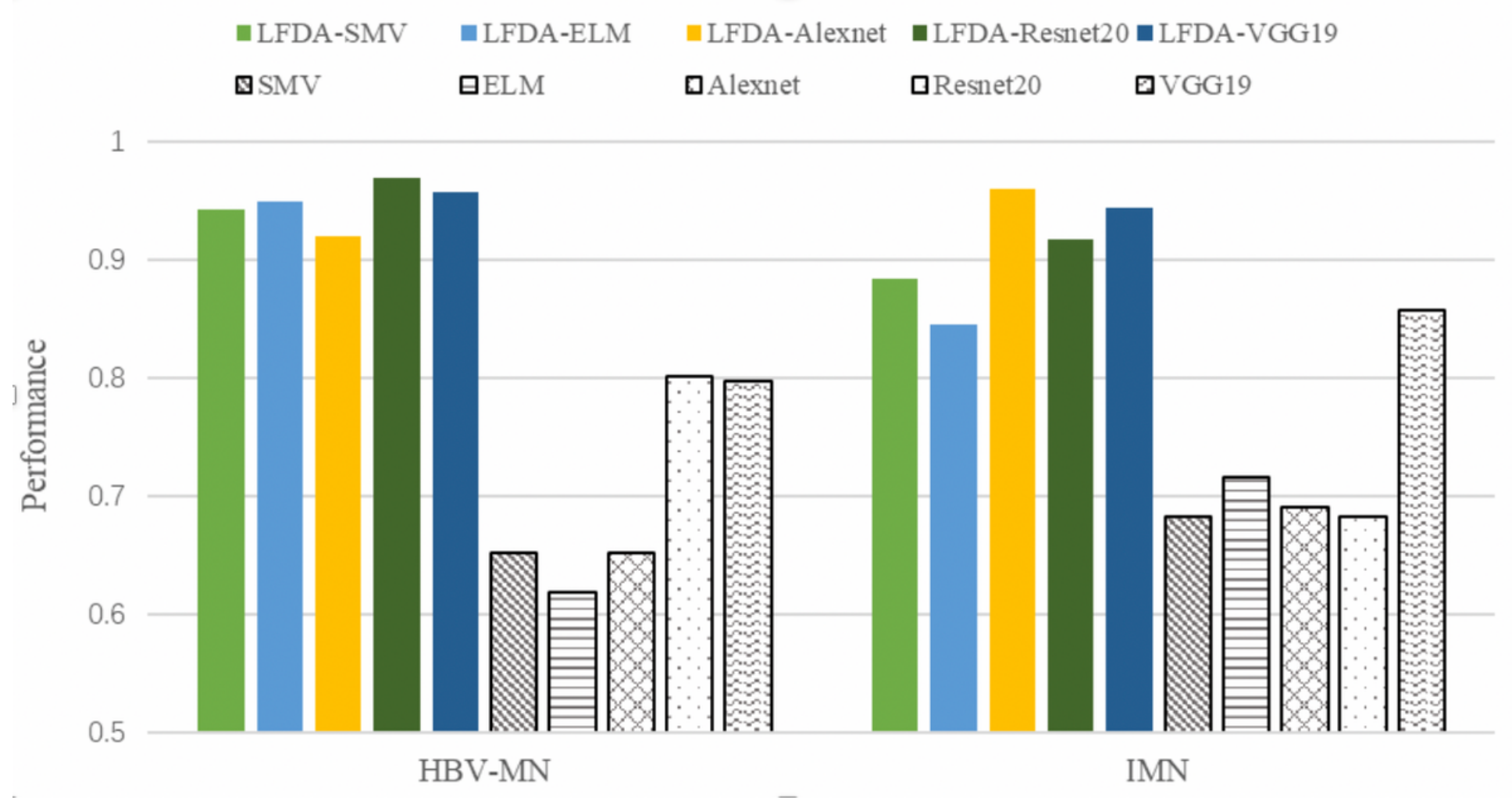

Figure 5

the comparison of performance obtained by each approach -HBV-MN $\square \mathrm{IMN}$ \# Accuracy

1

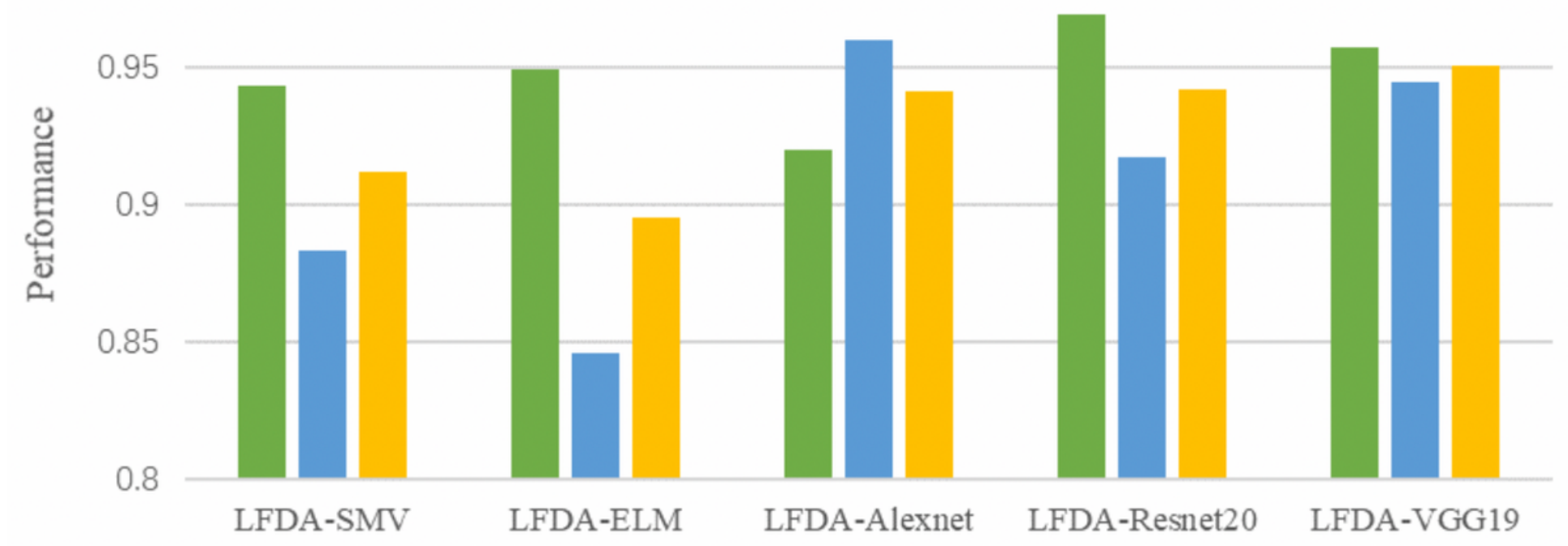

Figure 6

the overall accuracy of each LFDA-DNNS 